\title{
Thesaurus and Metadata Alignment for a Semantic E-Culture Application
}

\author{
Anna Tordai \\ VU University \\ 1081a De Boelelaan \\ Amsterdam, Netherlands \\ atordai@cs.vu.nl
}

\author{
Borys Omelayenko \\ VU University \\ 1081a De Boelelaan \\ Amsterdam, Netherlands \\ b.omelayenko@cs.vu.nl
}

\author{
Guus Schreiber \\ VU University \\ 1081a De Boelelaan \\ Amsterdam, Netherlands \\ schreiber@cs.vu.nl
}

\begin{abstract}
As the Semantic Web gains momentum, so grows the interest in making knowledge kept in various repositories available. In this paper we describe a methodological approach for porting cultural repositories to the Semantic Web, focusing on the global picture of the required mappings and alignments. The approach consists of thesaurus conversion, meta-data schema mapping, meta-data value mapping, and thesauri alignment. It is derived from our experience collected in a number of conversions we have performed for the E-Culture project, and in this paper it is illustrated with specific examples from a specific conversion case study.
\end{abstract}

\section{INTRODUCTION}

This article reports on work aimed at explicating the knowledge and data from an existing data source and making it available in a distributed Web context. The context of this work is the MultimediaN E-Culture project $[4]^{1}$, a leading Semantic Web project that won the Semantic Web Challenge in 2006. The objective of this project is to create a large virtual collection of culturalheritage objects that supports semantic search. Metadata and vocabularies are represented in RDF/OWL. The project demonstrator (see the demonstrator at the project website) includes multiple vocabularies which are partially semantically aligned.

The objective of the present work is to describe a methodological approach to make a new collection part of the overall virtual collection. We propose a four-step process to convert the thesaurus and metadata such that these become an interoperable part of the virtual col-

${ }^{1}$ http://e-culture.multimedian.nl lection. We illustrate the process with examples of the Bibliopolis collection ${ }^{2}$. The collection consists of images related to book-printing, and range from photographs of publishing houses to illustrations of the printing process.

This paper builds on earlier conversions of metadata and thesauri. There are currently 5 collections and 6 thesauri that are part of the E-Culture demonstrator.

To represent the collections the project uses a specialization of Dublin Core for visual resources (all objects in the virtual collection are required to have an image as their data representation) as the guiding metadata scheme. This Dublin Core specialization is named the VRA Core ${ }^{3}$ scheme which follows the Dublin Core dumb-down principle (i.e. it is a proper specialization and does not contain extensions). Likewise, we model collection-specific metadata schemes as specializations of VRA.

For the representation of thesauri the project uses the SKOS Core Schema ${ }^{4}$. It was designed to support vocabulary interoperability and is currently undergoing standardization by the World-Wide Web Consortium W3C. SKOS has already been adopted by large organizations such as NASA.

Our ultimate goal is to provide a systematic approach to build a large Semantic Culture Web. This paper is a first step towards a systematic approach for making clear to heritage institutions what they need to do to make their collections an interoperable part of this Culture Web.

This paper is organized as follows. We discuss related work in Section 2. Then, we present our approach in Section 3 followed by a short presentation of the Bibliopolis data in Section 4. Next we devote four sections to describe our method composed of four activi-

\footnotetext{
${ }^{2}$ http://www.bibliopolis.nl/

${ }^{3}$ http://www.vraweb.org/

${ }^{4}$ http://www.w3.org/TR/swbp-skos-core-guide/
} 
ties: thesaurus conversion, metadata schema mapping, metadata mapping and thesaurus alignment. Finally we conclude this paper with a discussion in Section 9.

\section{RELATED WORK}

In the area of thesaurus conversion Miles et. al. [3] propose guidelines for migrating thesauri to the Semantic Web using the SKOS Core schema. They distinguish between standard and non-standard thesauri, and propose to preserve all information in the thesaurus by using sub-class and sub-property statements where necessary.

The work of Van Assem et. al. [5] is based on these guidelines, and they propose a three step method consisting of the analysis of the thesaurus, mapping to the SKOS schema and the creation of the conversion program. The case studies do show however that non standard thesauri are more difficult to convert completely as some features cannot be mapped to the SKOS schema.

The problem of interoperability between two collections has been discussed in [1]. Within the SIMILE project Butler et.al. report on the conversion and linkage of a visual works dataset and learning object dataset using XSLT. The first dataset was converted using the VRA schema and the second using Dublin Core, although non standard properties were created as extensions. Issues discussed ranging from the creation of URI's, to the dealing with hierarchical terms.

In [2] Hyvönen et. al. describe the MuseumFinland project encompassing multiple collections and ontologies. The collections of various Finnish museums and ontologies converted into RDF/OWL. The metadata of collections was transformed using a common term ontology while the ontologies form an additional semantic link between the collections and were further enhanced by manual editing and enrichment.

\section{APPROACH}

Within the E-Culture project we developed a process for converting datasets to an interoperable Semantic Web format based on our experiences with several datasets (see the E-Culture demonstrator for details). The method defines four activities needed to convert a collection to a Semantic E-Culture Web application, summarized in Fig. 1:

- Thesaurus conversion, including thesaurus schema mapping. This step is a relatively well-researched area, e.g. [5], with SKOS being the default option for thesaurus schema.

- Metadata schema mapping. Here we are looking at generic schemas like Dublin Core and its specifications to the cultural domain, such as VRA.

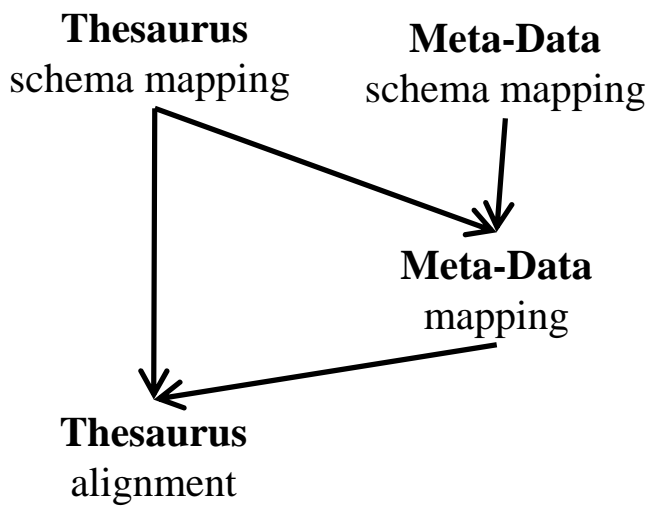

Figure 1: The four activities for converting a collection.

- Metadata mapping. At this step the data values are converted and looked up in the thesaurus or external vocabularies using the information extraction techniques. Data interpretation is also common here, especially for the data that does not directly fit the standard vocabularies.

- Thesaurus alignment. Here we align the thesaurus to external (standard) vocabularies with the ontology alignment techniques.

In the rest of the paper we describe these steps and illustrate it with examples from the Bibliopolis data.

\section{BIBLIOPOLIS DATA}

The Bibliopolis data from the Koninklijke Bibliotheek (KB), the National Library of the Netherlands, consists of two XML files: collection and thesaurus. The collection file contains the metadata of 1,645 images related to the printing of books and book illustrations. The thesaurus contains 1,033 terms used as keywords for indexing images. These two files are a part of the Bibliopolis website. Both the thesaurus and the metadata are bilingual (English and Dutch).

\section{Thesaurus}

The thesaurus contains core terms, augmented with their synonyms in plural, and variants of these terms in singular along with a descriptive note. Each record may also contain related, broader and narrower terms. Additionally, it contains some administrative data: initials of the record creator, the date of entry, and the date of modification. A sample XML element for the term university printer is shown in Fig. 2.

\section{Metadata}

The metadata forms the description of images related to book printing. The data consists of titles and descriptions of the objects, names of their creator(s) with signatures of their roles, such as a for author. The works 


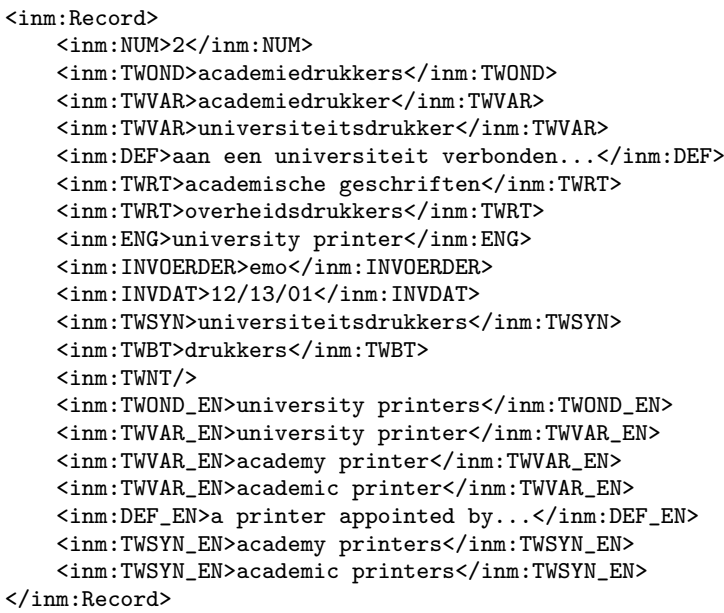

Figure 2: Thesaurus record for term University printer

are also classified according to the technique used, their type, and a library classification of the subject matter. The metadata includes copyright information, measurements and other administrative information. An example collection object plus corresponding metadata is shown in Fig. 3.

\section{THESAURUS CONVERSION}

Thesaurus schema mapping and conversion is a relatively well-researched area. In our work we used the method for thesauri conversion proposed by van Assem [5]. As of the thesaurus schema, we use SKOS within the E-Culture project.

Mapping the Bibliopolis thesaurus turned out to be relatively straightforward. Table 1 shows the details of the mapping of the thesaurus representation in Fig. 2 to SKOS. Two XML elements were not converted, as they contained bookkeeping information and are not meant for public consumption. One XML element (see last column in the table) turned out to be a duplicate piece of information and was therefore omitted.

The creation of the URI deserves special mention. When creating an URI we derive it from the real term identifier followed by the disambiguation signature and the thesaurus version. For example, in the Bibliopolis case the real identifiers are stored in field TWOND (and not NUM that contains a file-specific index rather than the real term identifier), they are unambiguous, and we have a single version.

\section{METADATA SCHEMA MAPPING}

In this activity we map the original record fields (see Fig. 3) to a metadata schema. In the E-Culture project we use the VRA Core scheme which is a specialization
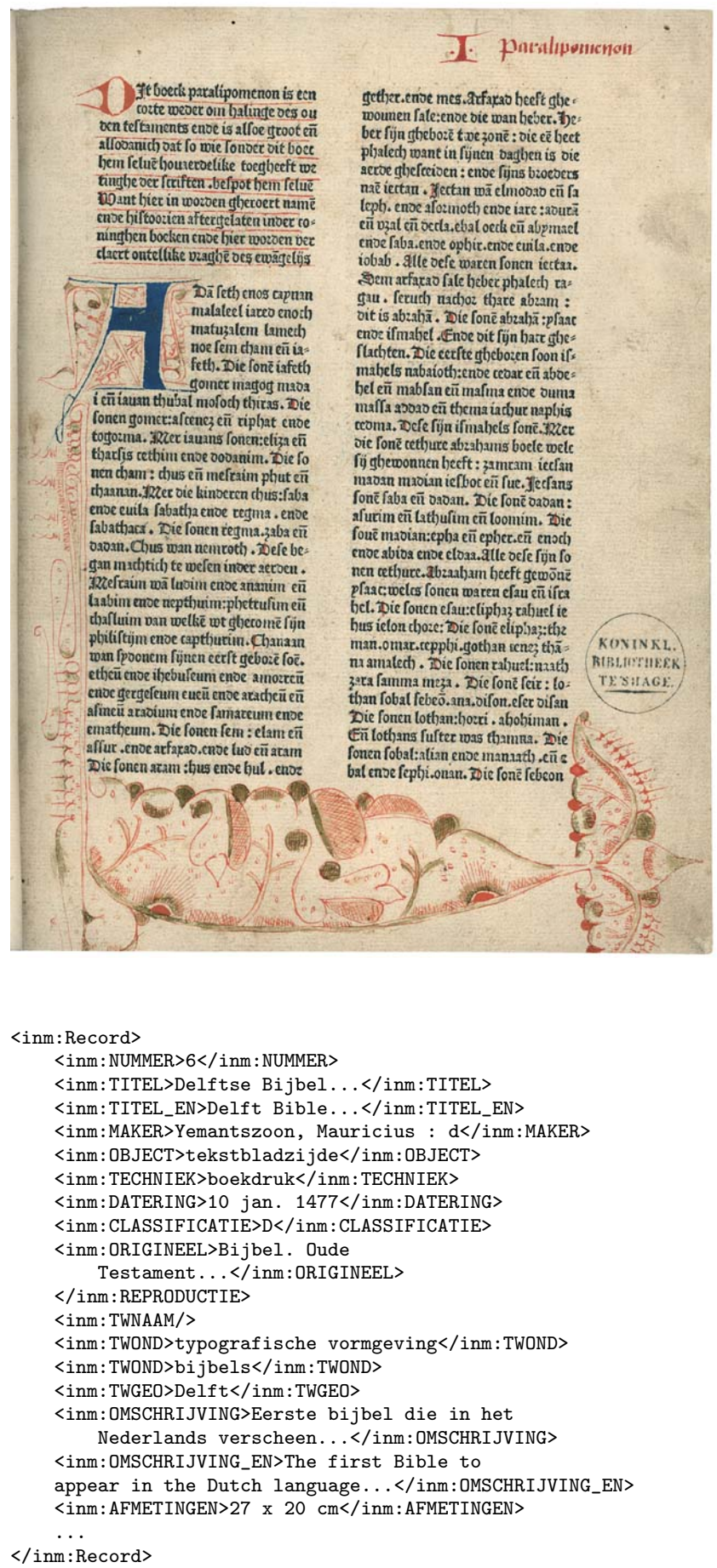

Figure 3: A fragment of XML record of a Delft Bible dated 10 January 1477 , originated from Delft, classified with category 'bibles'. 


\begin{tabular}{|c|c|c|c|}
\hline Data Item & Function & Activity & Source and Target Property/Class \\
\hline NUM & Internal identifier & Create literal & $\begin{array}{l}\text { source: } 2 \\
\text { target: vra:location.refId "2"; }\end{array}$ \\
\hline TWOND & Preferred term in Dutch & $\begin{array}{l}\text { Create URI, literal and language } \\
\text { tag }\end{array}$ & $\begin{array}{l}\text { source: academiedrukkers } \\
\text { target: bp:academiedrukkers rdf:type skos:Concept ; } \\
\text { skos:prefLabel "academiedrukkers"@nl ; }\end{array}$ \\
\hline TWSYN & Synonym in Dutch & Create literal and language tag & $\begin{array}{l}\text { source: universiteitsdrukkers } \\
\text { target: skos:altLabel "universiteitsdrukkers"@nl ; }\end{array}$ \\
\hline TWVAR & $\begin{array}{l}\text { Term in singular form in } \\
\text { Dutch }\end{array}$ & Create literal and language tag & $\begin{array}{l}\text { source: academiedrukker } \\
\text { target: skos:altLabel "academiedrukker"@nl ; }\end{array}$ \\
\hline $\mathrm{DEF}$ & Definition in Dutch & Create literal and language tag & $\begin{array}{l}\text { source: aan een universiteit verbonden... } \\
\text { target: skos:definition "aan een universiteit verbon- } \\
\text { den..."@nl; }\end{array}$ \\
\hline TWBT & Broader term & $\begin{array}{l}\text { Look up concept URI and add } \\
\text { URI }\end{array}$ & $\begin{array}{l}\text { source: drukkers } \\
\text { target: skos:broader bp:drukkers }\end{array}$ \\
\hline TWNT & Narrower term & $\begin{array}{l}\text { Look up concept URI and add } \\
\text { URI }\end{array}$ & $\begin{array}{l}\text { source: narrower term } \\
\text { target: skos:narrower bp:narrower_term ; }\end{array}$ \\
\hline TWRT & Related term & $\begin{array}{l}\text { Look up concept URI and add } \\
\text { URI }\end{array}$ & $\begin{array}{l}\text { source: overheidsdrukkers } \\
\text { target: skos:related bp:overheidsdrukkers ; }\end{array}$ \\
\hline TWOND_EN & $\begin{array}{l}\text { Preferred term in En- } \\
\text { glish }\end{array}$ & Create literal and language tag & $\begin{array}{l}\text { source: university printers } \\
\text { target: skos:prefLabel "university printers"@en ; }\end{array}$ \\
\hline TWSYN_EN & Synonym in English & Create literal and language tag & $\begin{array}{l}\text { source: academy printers } \\
\text { target: skos:altLabel "academy printers"@en ; }\end{array}$ \\
\hline TWVAR_EN & $\begin{array}{l}\text { Term in singular form in } \\
\text { English }\end{array}$ & Create literal and language tag & $\begin{array}{l}\text { source: university printer } \\
\text { target: skos:altLabel "university printer"@en ; }\end{array}$ \\
\hline DEF_EN & Definition in English & Create literal and language tag & $\begin{array}{l}\text { source: a printer appointed by... } \\
\text { target: skos:definition "a printer appointed by..."@en ; }\end{array}$ \\
\hline$\overline{\mathrm{ENG}}$ & $\begin{array}{lll}\text { English } & \text { translation } & \text { of } \\
\text { term }\end{array}$ & $\begin{array}{l}\text { Not converted; duplicate infor- } \\
\text { mation }\end{array}$ & source: university printer \\
\hline INVOERDER & Entered by & $\begin{array}{l}\text { Not converted: not part of re- } \\
\text { quirements }\end{array}$ & source: emo \\
\hline INVDAT & Date of entry & $\begin{array}{l}\text { Not converted: not part of re- } \\
\text { quirements }\end{array}$ & source: $12 / 13 / 01$ \\
\hline
\end{tabular}

Table 1: Mapping thesaurus data to SKOS

of Dublin Core ${ }^{5}$ for visual resources (our target type of resources).

Before mapping to the schema we analyze the metadata (including examination of any additional documentation, websites, and interviews with experts). The meaning of the fields needs to be understood to find a correct correspondence within the target schema. The first impression of the meaning of a field might be misleading. For example, the TWGEO field was initially mapped to vra:location, i.e., the DC/VRA element indicating where the work was created. However, the documentation showed that the field actually gives information about the location related to the subject, and not the creation place. We finally used the VRA Core v4 element vra:subject.geographicPlace, which gives the correct interpretation. This element is a subproperty of DC/VRA subject.

An important additional consideration is that certain records or fields may contain confidential or administrative information such as acquisition or bookkeeping information. For example, the amount for which an object is insured should not be publicly visible. This situation did not occur with the Bibliopolis data.

Table 2 shows an overview of the mapping from the XML record fields to a VRA metadata schema with

\footnotetext{
$\overline{{ }^{5} \text { http://dublincore.org/ }}$
}

examples. Here we face two situations. First, in the simplest case, there is a exact semantic match between an original field and a VRA field. Second, if this is not the case, the field should be specified as a specialization of an existing VRA element. In the Bibliopolis case this occurs with the ORIGINAL ${ }^{6}$, REPRODUCTION and CLASSIFICATION fields. The first two are specific "titles", the third one is a specific "subject" description. In Table 2 we see that the RDF/OWL specification contains property definitions in the Bibliopolis namespace (bp:) paired with a statement about the subproperty relationship with a VRA element.

One field requires some deeper study. The MAKER field not only contains the creator of the work, but also a character indicating the role that the person played in creating the work. As shown in the example record in Fig. 3 the MAKER field has the value Yemantszoon, Mauricius : d, where "d" stands for "drukker", Dutch for "printer". To preserve the roles of the creators we specialize the VRA property vra:creator with the properties that correspond to the roles found in the Bibliopolis data. This resulted in a set of $\mathrm{RDF} / \mathrm{OWL}$ definitions such as:

bp:drukker rdfs:subPropertyOf vra:creator bp:origineel rdfs:subPropertyOf vra:title bp:reproductie rdfs:subPropertyof vra:title

${ }^{6}$ For readability we use the English in the text, in cases where it is close to the Dutch equivalent ("original" vs. "origineel") 


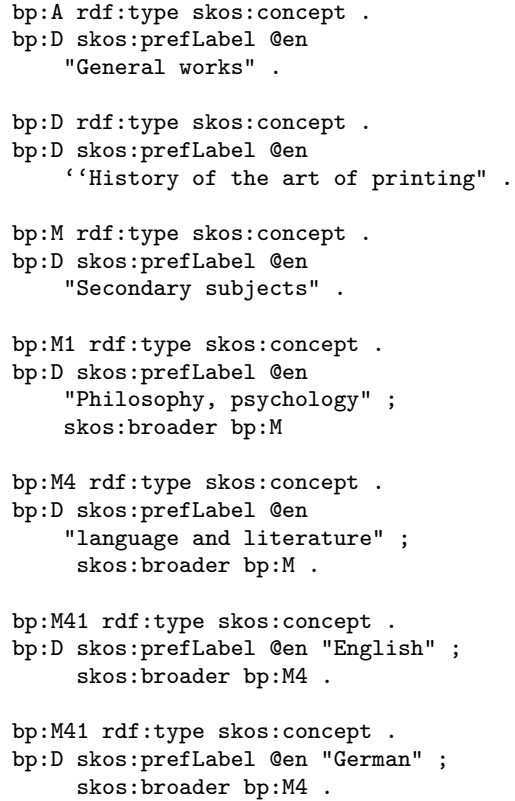

We distinguish six possibilities for value mapping, in decreasing order of priority:

1. Map a field value to a concept from the in-house vocabulary.

2. Map a field value to a concept in a newly-created vocabulary that was implicitly present in the original data.

3. Map a field value to a concept from another vocabulary

4. Map a field value to a concept that is a local extension of another vocabulary

5. Map a field value to a resource meant for future semantic extensions.

6. If none of the above is possible/desirable, create a literal for the field.

We illustrate each of these possibilities with an example from the Bibliopolist case.

\section{Mapping to an in-house vocabulary}

The values of the field TWOND represent thesaurus concepts. The RDF/OWL representations contains the corresponding URIs of these entries in the Bibliopolis thesaurus. As an aside, it is interesting to note that the records refer to the (unique) Dutch text label of the concept and not to the concept identifier. This is relevant information for the choice of the URI naming scheme for vocabulary concepts (cf. Section 5 ).

\section{Mapping to an implied vocabulary}

In Table 2 we see the value "D" for the field CLASSIFICATIE. Further analysis revealed that these single-letter

Figure 4: RDF specification (in N3 notation) of some sample classification concepts. The " $M$ " concept is the top concept of a BT/NT hierarchy

values actually represent a small vocabulary for librarytype classifications of the subject. This information is not part of the XML data, but is only shown on the website of Bibliopolis. This classification vocabulary has also some broader/narrower relations. We represented this vocabulary using the SKOS template and mapped the field values to concepts from this vocabulary.

The RDF example in Fig. 4 shows the SKOS specification of a subset of such classification subjects, including the $\mathbf{D}$ concept. The $\mathbf{M}$ concept ("secondary subjects") has a hierarchical substructure.

\section{Mapping to an existing vocabulary}

The values of TECHNIQUE and OBJECT fields were mapped to the Getty Art and Architecture Thesaurus (AAT) and the value of TWGEO to the Thesaurus of Geographical Names (TGN). Table 3 shows the number concepts occurring in the metadata, and the number that was mapped to the existing vocabularies. The values of these fields form a local vocabulary. The mapping of vocabulary to vocabulary is an alignment problem which is discussed further in Section 8.

\section{Mapping to a local extension}

When not all terms can be mapped to existing vocabularies, we create extensions. In the Bibliopolis data there were a small number of unmapped values of field TECHNIQUE (13) and of field OBJECT (5). We extend the AAT by adding the leftover terms to some part of 


\begin{tabular}{|c|c|c|c|}
\hline Data Item & Function & Activity & Source and Target Property/Class \\
\hline NUMMER & Record Id & $\begin{array}{l}\text { Create URI and additional pro- } \\
\text { ject specific triples (\&vra;Work) }\end{array}$ & $\begin{array}{l}\text { source: } 6 \\
\text { target: bp:6 rdf:Type vra:Work. }\end{array}$ \\
\hline TITEL & Title in Dutch & Create literal and language tag & $\begin{array}{l}\text { source: Delftse Bijbel... } \\
\text { target: vra:title "Delftse Bijbel..."@nl ; }\end{array}$ \\
\hline TITEL_EN & Title in English & Create literal and language tag & $\begin{array}{l}\text { source: Delft Bible... } \\
\text { target: vra:title "Delft Bible..." @en ; }\end{array}$ \\
\hline MAKER & $\begin{array}{l}\text { Creator and his marker } \\
\text { for role }\end{array}$ & $\begin{array}{l}\text { Extract name and role marker, } \\
\text { create URI and label for name } \\
\text { and convert marker to role, } \\
\text { create role as subproperty of } \\
\text { vra:creator }\end{array}$ & $\begin{array}{l}\text { source: Yemantszoon, Mauricius : d (d stands for } \\
\text { drukker meaning printer) } \\
\text { target: bp:drukker bp:Yemantszoon_Mauricius ; } \\
\text { bp:Yemantszoon_Mauricius rdf:type ulan:person ; } \\
\text { rdfs:label "Yemantszoon Mauricius". }\end{array}$ \\
\hline OBJECT & Object type & $\begin{array}{l}\text { Map to AAT or create local ex- } \\
\text { tension to AAT and mapping }\end{array}$ & $\begin{array}{l}\text { source: tekstbladzijde (text page) } \\
\text { target: vra:type bp:object_tekstbladzijde ; } \\
\text { bp:tekstbladzijde rdf:type skos:concept . } \\
\text { skos:prefLabel "tekstbladzijde"@nl ; } \\
\text { skos:broader AAT:pages ; }\end{array}$ \\
\hline TECHNIEK & Technique used & $\begin{array}{l}\text { Map to AAT or create local ex- } \\
\text { tension to AAT and mapping }\end{array}$ & $\begin{array}{l}\text { source: boekdruk (book printing) } \\
\text { target: vra:technique bp:techniek_boekdruk ; } \\
\text { bp:boekdruk rdf:type skos:concept . } \\
\text { skos:prefLabel "boekdruk"@nl ; } \\
\text { skos:broader AAT:printing; }\end{array}$ \\
\hline DATERING & Date & Interpret and filter data & $\begin{array}{l}\text { source: } 10 \text { jan. } 1477 \\
\text { target: vra:date "10-01-1477" }\end{array}$ \\
\hline $\begin{array}{l}\text { ORIGINEEL or RE- } \\
\text { PRODUCTIE }\end{array}$ & $\begin{array}{l}\text { Title of the original } \\
\text { or reproduction (book) } \\
\text { containing the image }\end{array}$ & $\begin{array}{l}\text { The title, author, date, place } \\
\text { and page number can be ex- } \\
\text { tracted) }\end{array}$ & $\begin{array}{l}\text { source: Bijbel. Oude Testament... } \\
\text { target: bp:origineel "Bijbel. Oude Testament..." @en } \\
\text {; }\end{array}$ \\
\hline CLASSIFICATIE & $\begin{array}{l}\text { Classification of the } \\
\text { work in librarian terms } \\
\text { using a code }\end{array}$ & $\begin{array}{l}\text { Interpret code, Create URI with } \\
\text { code, use interpretation as la- } \\
\text { bel keep identifier and create re- } \\
\text { source }\end{array}$ & $\begin{array}{l}\text { source: D (code interpreted as History of book print- } \\
\text { ing) } \\
\text { target: bp:classificatie bp:D ; }\end{array}$ \\
\hline TWNAAM & $\begin{array}{l}\text { Person used as subject } \\
\text { for work }\end{array}$ & Interpret name and create URI & $\begin{array}{l}\text { source: John Do } \\
\text { target: vra:subject.personalName bp:John_Do ; } \\
\text { bp:John_Do rdf:type ulan:person ; } \\
\text { rdfs:label "John Do". }\end{array}$ \\
\hline TWOND & $\begin{array}{l}\text { Thesaurus term used as } \\
\text { subject }\end{array}$ & Create mapping to thesaurus & $\begin{array}{l}\text { source: typografische vormgeving } \\
\text { target: vra:subject bp:typografische_vormgeving ; }\end{array}$ \\
\hline TWGEO Delft & $\begin{array}{l}\text { Place used as subject for } \\
\text { work }\end{array}$ & $\begin{array}{l}\text { Create mapping to TGN where } \\
\text { possible or keep literal with lan- } \\
\text { guage tag }\end{array}$ & $\begin{array}{l}\text { source: Delft } \\
\text { target: vra:subject.geographicPlace tgn:7006804; }\end{array}$ \\
\hline $\begin{array}{l}\text { OMSCHRIJVING } \\
\text { or OMSCHRIJV- } \\
\text { ING_EN }\end{array}$ & $\begin{array}{l}\text { Dutch or English de- } \\
\text { scription }\end{array}$ & Create literal and language tag & $\begin{array}{l}\text { source: Eerste bijbel die... } \\
\text { target: vra:description "Eerste bijbel die..."@nl ; }\end{array}$ \\
\hline AFMETINGEN & Size of the work & Create literal & $\begin{array}{l}\text { source: } 27 \times 20 \mathrm{~cm} \\
\text { target: vra:measurements.dimensions " } 27 \times 20 \mathrm{~cm} \text { " }\end{array}$ \\
\hline
\end{tabular}

Table 2: Part of the Bibliopolis metadata with examples, function and RDFS property/classes

the vocabulary if possible. For instance, the technique boekdruk (book printing) is not part of AAT but is a special kind of printing technique, therefore the AAT concept printing is selected as broader term. We use the SKOS template to represent the extension. To create a unique URI for our extension we add the field name to the field value resulting in \&bp; techniek_boekdruk, which is part of the bp: namespace. The reason for this is that the values of TECHNIQUE and OBJECT sometimes coincide, for example, foto is a technique as well as an object type.

\section{Mapping to a typed resource}

We create resources from field values that will be used for future semantic extensions. In general these may be names of organizations or persons, places, cultures or historical periods. In Bibliopolis the values of MAKER and TWNAAM contain person names. These names can possibly be linked to United List of Artist Names (ULAN) vocabulary. We create resources out of these names with URI's in the bp: namespace removing in- valid characters and spaces. The concepts are of type ulan:person and the human readable label contains the name.

\section{Mapping to a literal}

Pieces of text such as titles and descriptions are converted to literals. An important guideline is to specify the language of the text in language tags. In Bibliopolis we are dealing with both Dutch and English language labels in the TITLE and DESCRIPTION fields.

\section{THESAURUS ALIGNMENT}

The local thesaurus and the vocabularies containing techniques, object types and locations extracted from the data during the metadata conversion process need to be aligned with the standard vocabularies.

We aligned the Bibliopolis thesaurus to AAT by syntactically matching the Dutch skos:prefLabel to the Dutch translation of AAT preferred terms and mapped 209 concepts out of 1033 as presented in Table 3. 


\begin{tabular}{|c|c|c|c|c|c|}
\hline \multirow[t]{2}{*}{ Source Data } & \multirow[t]{2}{*}{ Vocabulary } & \multicolumn{2}{|c|}{ Terms } & \multicolumn{2}{|c|}{ Instances } \\
\hline & & Mapped & Total & Mapped & Total \\
\hline Thesaurus & $\overline{\text { AAT }}$ & 209 & 1033 & - & - \\
\hline $\begin{array}{l}\text { Metadata } \\
\text { technique }\end{array}$ & $\overline{\text { AAT }}$ & 15 & 28 & 1332 & 1468 \\
\hline $\begin{array}{l}\text { Metadata } \\
\text { object type }\end{array}$ & AAT & 14 & 19 & 978 & 1507 \\
\hline $\begin{array}{l}\text { Metadata } \\
\text { subject } \\
\text { place }\end{array}$ & TGN & 32 & 69 & 349 & 480 \\
\hline
\end{tabular}

Table 3: Mappings between the Bibliopolis data and other vocabularies

Then, we need to identify the relation between the matched terms. The OWL owl:sameAs relation is typically an overstatement that we try to avoid, as ambiguity is quite common. The SKOS Mapping Vocabulary specification ${ }^{7}$ was created for the purpose of linking thesauri to each other. It specifies relationships such as skos:exactMatch, skos:broadMatch, skos:narrowMatch and more for aligning vocabularies. For this alignment the mappings are still based on the lexical match of term labels, that corresponds to the relation skos:exactMatch.

Geographical names, however, form a frequent exception. With a few additional simple restrictions, a lexical match gives enough confidence to generate a semantic match as strong as owl:sameAs. As an example, a mapping to "Paris", known that it is a city in France, can be made with owl: sameAs.

\section{DISCUSSION}

Interoperability is becoming one of the key issues in the open Web world. Many research programs, such as the IST program of the EU, have interoperability high on the agenda. However, real interoperability between collections is still scarce. Until now, many approaches have focused on interoperability as a problem between two collections.

In this paper we take a different approach. We assume a multitude of collections will become part of the interoperable space; the activities we present can to a large extent be carried out by studying an individual collection. Mapping to existing other vocabularies requires knowledge of other components, but there is no need for these to be complete. For vocabulary alignment the adage "a little semantics goes a long way" 8 holds. Also, one should not view this as a one-shot thing. Metadata and vocabularies change, so extensions will take place at regular intervals in time. This also means that tool support should be in place to support this process, allowing updates to be generated semi-automatically, similar to the AnnoCultor ${ }^{9}$ that is being currently developed within the E-Culture project.

\footnotetext{
${ }^{7}$ http://www.w3.org/2004/02/skos/mapping/spec/

${ }^{8}$ quote from J. Hendler

${ }^{9}$ http://annocultor. sourceforge.net/
}

For the E-Culture virtual collection we have now carried out this process a number of times. This paper should be viewed as a post-hoc rationalization of this work. Our goal is to provide a set of methods and tools that allow collection owners (museums, archives) to carry out this process. Cultural-heritage institutions are now often bound to closed content management systems; the "three-O" paradigm (open access, open data, open standards) is gaining support, but we have to provide the collections owners with the necessary support facilities.

We see two potential weaknesses of this work. Firstly, our process still requires much more tool support. In particular for vocabulary alignment we need to explore how existing tools, such as the ones participating in the OAEI contest, perform on this data set. Our current work is still to much based on hand work and only uses simple syntactic tools.

Secondly, the use of Dublin Core as "top-level ontology" for the structure as metadata can also be perceived as a risk. What if the collection has metadata fields that fit with none of the DC elements? However, this was not a problem in either of these six collections. For the moment it seems Dublin Core is indeed a key resource in information interoperability. However, it is a challenge to construct reasoners that make use of the collectionspecific specializations.

This article does not show the actual added value of the converted collection content. For this the readers are encouraged to visit the E-Culture online demonstrator, which contains the Bibliopolis data.

\section{ACKNOWLEDGMENTS}

We are grateful to our colleagues from the Multimedian E-Culture team: Alia Amin, Lora Aroyo, Victor de Boer, Lynda Hardman, Michiel Hildebrand, Marco de Niet, Annelies van Nispen, Marie France van Orsouw, Jacco van Ossenbruggen, Annemiek Teesing, Jan Wielemaker and Bob Wielinga. Theproject is a collaboration between the Free University Amsterdam, the Centre of Mathematics and Computer Science (CWI), the University of Amsterdam, Digital Heritage Netherlands (DEN) and the Netherlands Institute for Cultural Heritage (ICN). The MultimediaN project is funded through the BSIK programme of the Dutch government.

\section{REFERENCES}

[1] M. H. Butler, J. Gilbert, A. Seaborne, and K. Smathers. Data conversion, extraction and record linkage using xml and rdf tools in project simile. Technical report, Digital Media Systems Laboratory and HP Laboratories, August 2004.

[2] E. Hyvönen, E. Mäkelä, M. Salminen, A. Valo, K. Viljanen, S. Saarela, M. Junnila, and S. Kettula. Museumfinland-finnish museums on 
the semantic web. Web Semantics: Science, Services and Agents on the World Wide Web, 3(2-3):224-241, October 2005.

[3] A. J. Miles, N. Rogers, and D. Beckett. Migrating thesauri to the semantic web - guidelines and case studies for generating rdf encodings of existing thesauri.

[4] G. Schreiber, A. Amin, M. van Assem, V. de Boer, L. Hardman, M. Hildebrand, L. Hollink, Z. Huang, J. van Kersen, M. de Niet, B. Omelayenko, J. van Ossenbruggen, R. Siebes, J. Taekema, J. Wielemaker, and B. J. Wielinga. Multimedian e-culture demonstrator. In I. F. Cruz, S. Decker, D. Allemang, C. Preist, D. Schwabe, P. Mika, M. Uschold, and L. Aroyo, editors, International Semantic Web Conference, volume 4273 of Lecture Notes in Computer Science, pages 951-958. Springer, 2006.

[5] M. van Assem, V. Malaisé, A. Miles, and G. Schreiber. A method to convert thesauri to SKOS. In Y. Sure and J. Domingue, editors, ESWC, volume 4011 of Lecture Notes in Computer Science, pages 95-109. Springer, 2006. 